\title{
Respuesta al factor liberador en niños con deficiencia de hormona de crecimiento
}

\author{
Hemán García B. ${ }^{1}$; Ricardo Silva A. ${ }^{1}$; Angélica Boric $S^{2}{ }^{2}$; \\ Alejandra Avila $\mathrm{A}^{3}{ }^{3}$; Medardo Burgueño A. ${ }^{4}$; Fernando Cassorla $\mathrm{G}^{3}$ \\ Growth hormone response to releasing factor \\ in growth hormone deficient children
}

\begin{abstract}
Growth hormone releasing factor $(G R F)$, is a peptide of 44 aminoacids produced in the hvpothalamus which stimulates the secretion of growth hormone $(\mathrm{GH})$ by the pituitary gland. This factor was administered to 21 prepubertal chiddren with growth hormone deficiency 112 bovs and 9 gir|sl. with mean chronological age of $8.9 \pm$ 3.5 vears, and mean bone age $5.6 \pm 2.6$ vears. The diagnosis of growth hormone deficiency was based upoา a $\mathrm{GH}$ response under $7 \mathrm{ng} / \mathrm{ml}$ to two stimulation tests, height 2 S.D. or greater below the mean, and growth velocity below $4.5 \mathrm{~cm}$ per year. A positive response to the GRF test was defined as an increment in $\mathrm{GH}$ levels greater than 4 times the coefficient of variation of the assav used There was a positive response to GRF in 13 of 21 patients $(62 \%)$. The peak $\mathrm{GH}$ level in response to $\mathrm{GRF}$ was $7.2 \pm 10.8 \mathrm{ng} / \mathrm{ml}$, and it was observed between 5 and $30 \mathrm{mi}-$ nutes after GRF administration. The positive response to GRF in $62 \%$ of our growth hormone deficient patients suggests that most of theem have a hypothalamic defect and not a pituitary disorder. These patients might benefit from long-term treatment with GRF.
\end{abstract}

(Key words: growth disorders, growth hormane, pituitary, somatotropin, growth hormone, releasing factor )

La hormona de crecimiento (GH) o somatotropina es secretada en forma pulsátil por la hipófisis. La regulación de los pulsos de $\mathrm{GH}$ es la re. sultante de la liberación intermitente de dos péptidos producidos en el hipotálamo: uno que estimula la secreción de la hormona de crecimiento, llamado factor liberador u hormona liberadora de somatotropina (GRF), y otro que la inhibe, denominado somatostatina, cuya interrelación produce estimulación o inhibición

1. Instituto de Investigaciones Materno-Infantil, Facultad de Medicina, Universidad de Chile, Hospital San Borja-Arriarán.

2. Bioquímica. Instituto de Investigaciones MaternoInfantil, Facultad de Medicina, Universidad de Chile, Hospital San Borja-Arriarán.

3. Enfermera. Instituto de Investigaciones MaternoInfantil, Facultad de Medicina, Universidad de Chile, Hospital San Borja-Arriarán.

4. INTA, Instituto de Nutrición y Tecnología de los Alimentos, Universidad de Chile.

Proyecto financiado a través de FONDECYT 91 . 1020 . neta sobre los somatotropos hipofisiarios, que son las células encargadas de la sintesis y secreción de GH (figura 1).

La deficjencia de $\mathrm{GH}$ obedece a diferentes causas, que pueden afectar al hipotálamo, la hipófisis o ambos. El único tratamiento disponible actualmente para estos pacientes es la administración de somatotropina, que tiene un costo muy elevado, por lo que es poco accesible para la mayoria de nuestros pacientes. Cabe mencionar que la talla final de nitos con deficiencia, no tratados, es de alrededor de 4 desviaciones estándar bajo la media, lo que refleja la importancia de tratar oportunamente a estos pacientes.

La hormona liberadora es un péptido hipotalámico de 44 aminoácidos que ha sido sintetizado recientemente. Se ha estudiado la respuesta al estímulo con factor liberador en voluntarios adultos normales, en niffos y adolescentes normales $y$ en niffos con deficiencia de $\mathrm{GH}$. Un porcentaje siguificativo de pacientes con deficiencia de GH responde al estímulo con factor liberador, lo que sugiere que la mayor parte de estos pacientes tiene una falla en la señal hi- 


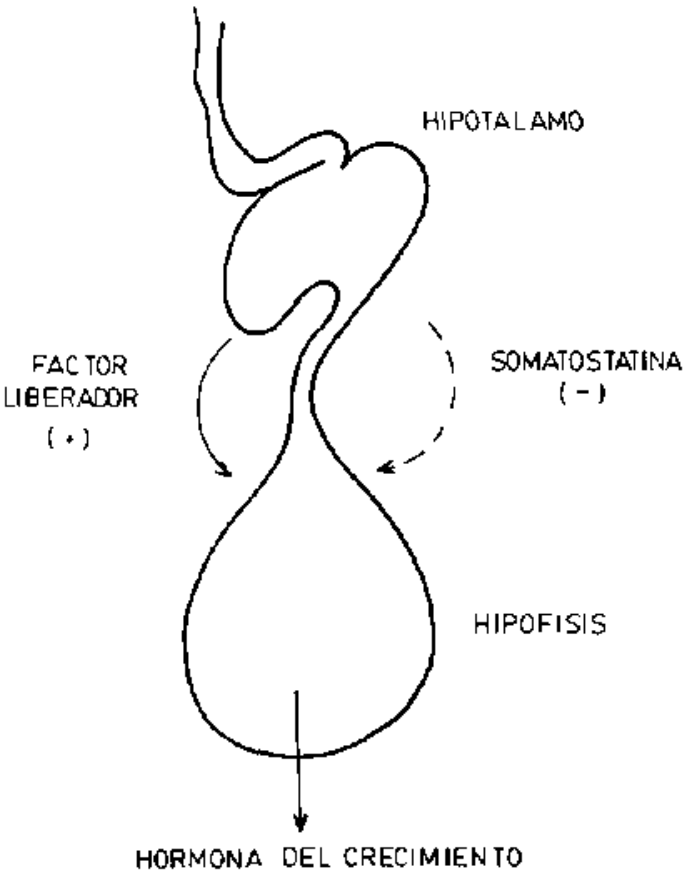

Figura 1: Regulación de la secreción de hormona de crecimiento. El factor liberador (GRF) estimula, mientras que la somatostatina inhibe su secteción.

potalámica que controla la secreción de $\mathrm{GH}$, más que un defecto hipofisiario' ${ }^{1-7}$.

En base a estos resultados, el factor liberador ha sido usado en forma crónica como agente terapéutico en algunos pacientes con deficiencia de $\mathrm{GH}^{8,9}$, en especial en casos que han mostrado respuesta positiva al estímulo con el mismo. Por lo tanto, la prueba con la hormona liberadora se ha empleado para identificar los pacientes en quienes podría considerarse esta alter. nativa terapéutica.

El objetivo del presente trabajo fue estudiar la respuesta de $\mathrm{GH}$ a la estimulación con factor $\mathrm{b}$ berador en un grupo de niños prepuberales con deficiencia de hormona de crecimiento.

\section{Material y Métodos}

Se administró factor liberador a 21 niños prepuberales (12 mujeres) con deficiencia de hormona de crecimiento, que no habían recibido tratamiento antes del estudio. Todos los pacientes cumplieron con los siguien. tes criterios: talla $<2$ DE bajo la media de las tablas del NCHS ( 2 SD: $-4,2 \pm 1,6$ ), velocidad de crecimiento inferior a $4,5 \mathrm{~cm} /$ año, respuesta inferior a $7 \mathrm{ng} / \mathrm{ml}$ a dos pruebas de est ímulo para GH (con clonidina o L-dopa e insulina) y ausencia de otra enfermedad. El déficit de $\mathrm{GH}$ fue considerado idiopático en 20 pacientes y secundario a craneofaringioma en uno. La edad ctonológica promedio fue de $8,9 \pm 3,5$ años y la edad ósea promedio fue de $5,6 \pm 2,6$ años. Las caracter ísticas clínicas de estos pacientes se muestran en la tabla 1.

El peso de nacimiento promedio fue te $3222 \pm$ $928 \mathrm{~g}$. El antecedente de asfixia perinatal o parto traumático estuvo presente en dos de los pacientes estudiados. El examen físico no demostró la presencia de estigmas genéticos en ninguno de ellos. Ninguno de los pacientes mostraba signos de desarrollo puberal fausencia de desarrollo mamario y vello pubiano en las niñas; volumen testiculat inferior a $3 \mathrm{ml}$ en los varones). En todos se realizó hemograma, perfil bioquímico, carotinemia, estudio de función tiroidea (T4 y TSH), cortisol y cromatina nuclear. Excepto por un varón con hipotiroidismo confirmado y tratado con L-tiroxina antes de la prueba con factor liberador, los dernás estaban libres de otras deficiencias hormonales. En cinco de nueve mujeres se realizó cariograma. Todos estos exámenes resultaron normales. En 14 de $21 \mathrm{pa}$ cientes se efectuó tornografía axial computadorizada (TAC).

La hormona de crecimiento se determinó durante al menos dos pruebas de estimulo; una con clonidina (100 $\mu \mathrm{g}$ por $\mathrm{m}^{2}$ vía oral) o L-dopa (125 mg vía oral en ninos con peso < $10 \mathrm{~kg} \mathrm{y} 250 \mathrm{mg}$ si éste era mayor), y otra con insulina $(0,05$ a $0,1 \mathrm{u} \cdot \mathrm{kg}$ de peso ev). Ambas se efectuaton por la mañana, en ayunas, tomándose muestras para medición de $\mathrm{GH}$ a los $0 \cdot 15-30 \cdot 60$ 90 y 120 minutos.

La prueba con factor liberador también se realizó por la mañana, en ayunas. Consistió en la administración de GRF (GHRH 1-44 $\mathrm{NH}_{2}$, Bachem, Inc. Torranee, CA) en dosis de $1 \mu_{g}-\mathbf{k g}$ ev, con muestras para $\mathbf{G H}$ a los $0-5 \cdot 10 \cdot 15-30-45$ y 60 minutos

Para la medición de hormona de crecimiento en plasma se usó un radioinmunoensayo comercjal con segundo anticuerpo (DPC), cuyo coeficiente de variación intraensayo fue de $5 \%$ e interensayo de $7 \%$. Se definió como respuesta positiva a la prueba con factor liberador, un aumento en los niveles de hormona de crecimiento mayor de 4 veces el coeficiente de variación del RIA utilizado'.

\section{Resultados}

Se observó una respuesta positiva a la prueba de estímulo con factor liberador en 13 de $\operatorname{los} 21$ pacientes (62\%) (tabla 2). La respuesta máxima $(17,2 \pm 10,8 \mathrm{ng} / \mathrm{ml})$ a la prueba se obtuvo entre los 5 y $\mathbf{3 0}$ minutos en todos los pacientes (figura 2).

La TAC fue normal en 10 pacientes. En tres se demostró un aracnoidocele selar y en uno un craneofaringioma operado. En los tres pacientes con aracnojdocele selar la respuesta a la prueba con factor liberador fue positiva, mientras on el 
Tabla 1

Pincipales caracteristicas clínicas de los pacientes con defficit de somatotropina y respuesta máxima al est imulo con hormoni liberadora

\begin{tabular}{|c|c|c|c|c|c|}
\hline Paciente & Sexo & $\begin{array}{l}\text { Edad } \\
\text { (aภ̂̃os) }\end{array}$ & $\begin{array}{c}\text { Edad ósea } \\
\text { (años) }\end{array}$ & $\begin{array}{l}\text { Talla } \\
\text { Z DE }\end{array}$ & $\begin{array}{l}\text { GH máx } \\
(\mathrm{ng} / \mathrm{ml})\end{array}$ \\
\hline 1 & $\mathbf{M}$ & 3,2 & 2,0 & $-2,6$ & 20,0 \\
\hline 2 & $\mathrm{~F}$ & 3,4 & 2,2 & $-4,5$ & 3,6 \\
\hline 3 & $\mathbf{M}$ & 3,9 & 1,1 & $-7,3$ & 0,5 \\
\hline 4 & M & 4,1 & 3,1 & $-2,2$ & 1,5 \\
\hline 5 & $\mathbf{F}$ & 5,5 & 4,2 & $-6,0$ & 1,0 \\
\hline 6 & $M$ & 6.5 & 2,8 & $-6,5$ & 5.8 \\
\hline 7 & $\mathbf{M}$ & 72 & 3,4 & $-4,5$ & 16,5 \\
\hline$g$ & $\mathbf{M}$ & 73 & 3,6 & $-2,0$ & 25,9 \\
\hline 9 & $\mathbf{M}$ & 8,5 & 6,5 & $-3,1$ & 40,6 \\
\hline 10 & $F$ & 8,7 & 3,7 & $-5,3$ & 11,6 \\
\hline 11 & M & 9,0 & 7,0 & $-2,0$ & 153 \\
\hline 12 & $\mathbf{F}$ & 9.8 & 7,2 & $-3,9$ & 0,9 \\
\hline 13 & $\mathbf{M}$ & 10,1 & 6,6 & $-4,7$ & 1,6 \\
\hline 14 & $\mathrm{~F}$ & 10,3 & 7,8 & $-3,0$ & 30,0 \\
\hline 15 & $\mathrm{~F}$ & 11,6 & 8,6 & $-3,9$ & 1,0 \\
\hline 16 & M & 11,6 & 8,8 & $-2,3$ & 27,5 \\
\hline 17 & $\mathbf{F}$ & 11,7 & 8,8 & $-4,5$ & 9,2 \\
\hline 18 & $F$ & 11,8 & 5,9 & $-6,5$ & 1,6 \\
\hline 19 & M & 12,0 & 6,8 & $-3,4$ & 10,5 \\
\hline 20 & $\mathbf{M}$ & 135 & 7,5 & $-4,6$ & 1,0 \\
\hline 21 & $\mathbf{F}$ & 16,8 & 10,5 & $-6,1$ & 12,7 \\
\hline
\end{tabular}

GH: somatotropina,

Tabla 2

Respuesta a la prueba con hormora liberadora de somatotropina en pacientes con deficiencia de hormona de crecimiento

\begin{tabular}{crrrr}
\hline Pacientes & $\mathrm{n}$ & $*$ & $\begin{array}{c}\text { GH basal } \\
\text { (ng/ml) }\end{array}$ & $\begin{array}{c}\text { GH máximo } \\
\text { (ng/ml) }\end{array}$ \\
\hline GRF (+) & 13 & 62 & $1,3 \pm 1,1$ & $17,2 \pm 10,8$ \\
GRF (-) & 8 & 38 & $0,8 \pm 0,4$ & $1,1 \pm 0,4$ \\
\hline
\end{tabular}

GH : hormona de crecimiento.

GRF : factor liberador de hormona de crecimiento.

(t) : respuesta positiva.

(-) : respuesta negativa.

paciente con craneofaringioma no hubo respuesta.

No hubo efectos indeseables a la inyección de factor liberador. Los parámetros hemodinámicos se mantuvieron estables durante la prueba. Al finalizar ésta los pacientes fueron enviados a sus hogares, no refiriendo ningún problema posterior.

\section{Comentario}

Nuestro estudio demostró respuesta positiva al estímulo con factor liberador en 13 de 21 niños prepuberales con deficiencia de hormona de crecimiento $(62 \%)$. Ello sugiere que un porcentaje importante de estos pacientes podría beneficiarse con un tratamiento crónico con factor 


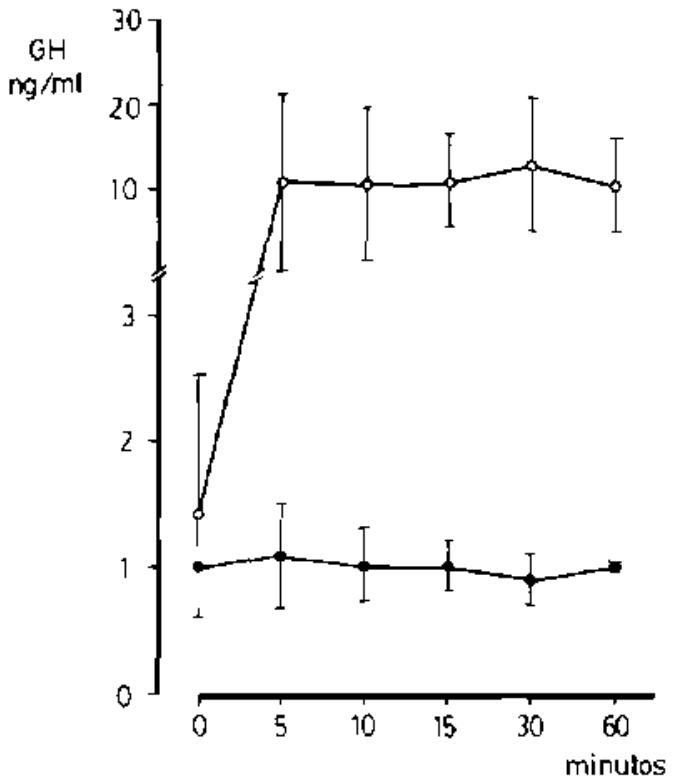

GRF $(+)=0 \longrightarrow n=13$

$\mathrm{GRF}(-)=\mathrm{n}=8$

Figura 2: Concentración sérica de somatotropina 5, $10,15,30$ y 60 minutos después de la administración de hormona liberadora en pacientes con respuestas positiva $(+)$ y negativa $(-)$.

liberador, lo que tiene implicaciones tanto médicas como económicas.

La existencia de un factor liberador de hormona de crecimiento había sido sospechada desde $1950^{1,2}$. Su identificación como un péptido de 44 aminoáciơos y su secuencia aminoacídica fue descubierta en 1982 por dos grupos de investigadores que estaban trabajando en forma independiente $\mathrm{e}^{3,4}$.

Diversos autores han descrito que la administración de factor liberador es capaz de estimular la liberación de GH en una proporción importante de pacientes $(40$ a $80 \%$ ) con déficit de $\mathrm{GH}^{5,6}$. Por otra parte, la respuesta de niños y adolescentes normales al factor liberador es bastante heterogénea ${ }^{2}$. Cabe mencionar que se ha descrito menor respuesta en pacientes con defíciencia de $\mathrm{GH}$ después de los 16 años de edad, Jo que podría estar reflejando el efecto de un período muy prolongado de atrofia de los somatotropos. De allí la importancia de considerar esta alternativa terapéutica lo más precozmente posible en el niño, ya que ello aumenta- ría la probabilidad de responder al tratamiento con factor liberador.

En la mayoria de nuestros pacientes estudia. dos confirmamos respuesta positiva al factor liberador (62\%), siendo la máxima al estímulo $(17,2 \pm 10,2 \mathrm{ng} / \mathrm{ml})$, aproximadamente dos tercios de la observada en niños prepuberales normales. Este nivel máximo se obtuvo antes de los 30 minutos en todos los pacientes con respuesta positiva. Esto sugiere que la prueba se podría abreviar, lo cual reduciría su costo.

Esta proporción de respuestas positivas en nifios con deficiencia de GH señala que ella es de naturaleza hipotalámica y que, por lo tanto, existe relativa indemnidad hipofisiaria en la mayor parte de estos casos. Es posible que estos pacientes pudieran beneficiarse de un tratamiento prolongado con factor liberador, el cual se está llevando a efecto en la actualidad en la mayoría de jos ąue atendemos en nuestro hospital.

Existe información sobre la utilídad terapéutica del factor liberador administrado en forma crónica a niños con deficiencia de $\mathrm{GH}^{10}$, cuyo tratamiento se encuentra actualmente restringido a la hormona de crecimiento, de alto costo, para lo cual el factor liberador representa una interesante alternativa. Considerando que su efecto $\mathrm{es} \mathrm{mediado} \mathrm{por} \mathrm{la} \mathrm{estimulación} \mathrm{de} \mathrm{la} \mathrm{hormo-}$ na de crecimiento endógena producida por la propia hipófisis del paciente, la eficacia de esta opción será dependiente del grado de respuesta hipofisiaria. En consecuencia, una respuesta positiva al estímulo con factor liberador tiene utilidad para identificar a aquellos pacientes en los cuales su empleo podría ser efectivo.

En conclusión, el factor liberador parece constituir una alternativa terapéutica promisoria para el tratamiento de niños con deficiencia de hormona de crecimiento. Actualmente diversas compañías farmacéuticas están tratando de producir una variante farmacéutica de depósito, cuyo uso eliminaría la necesidad de administrar el producto en forma frecuente, 10 que simplificaría su aplicación clínica.

\section{Resumen}

El factor liberador de hormona de crecimiento (GRF) es un péptido de 44 aminoácidos producido en el hipotálamo, que estimula la secre- 
ción de hormona de crecimiento (GH) por la hipófisis. Este factor fue administrado a $21 \mathrm{ni}$ nos prepuberales (12 varones y 9 mujeres) portadores de deficiencia de hormona de crecimiento. La edad cronológica promedio fue de $8,9 \pm 3,5$ años, y su edad ósea de $5,6 \pm 2,6$ años. El diagnóstico de deficiencia de $\mathrm{GH}$ se basó en una talla $2 \mathrm{DE}$ bajo la media, velocidad de crecimiento inferior a $4,5 \mathrm{~cm} / \mathrm{año}$, respuesta inferior a $7 \mathrm{ng} / \mathrm{ml}$ a 2 diferentes pruebas de estímulo para $\mathrm{GH}$, y ausencia de otras afecciones. La respuesta al estímulo con GRF fue definida co. mo positiva cuando los niveles de $\mathrm{GH}$ aumentaron por sobre 4 veces el coeficiente de variación del radioinmunoensayo utilizado, lo que se registró en 13 de los 21 pacientes $(62 \%)$. La respuesta máxima al GRF fue $17.2 \pm 10,8 \mathrm{ng} / \mathrm{ml}$ y se observó entre 5 y $30 \mathrm{~min}$ después de su administración. Los resultados positivos en $62 \%$ de los pacientes estudiados sugieren que sus defectos residen en el hipotálamo más que en la hipófisis. Estos niños se podrían beneficiar de un tratamien to a largo plazo con GRF.

(Palabras claves: Trastornos del crecimiento, hormona de crecimiento, pituitaria, hipófisis, somatotropina, factor liberador.)

\section{Agradecimientos}

A los médicos S. Aserio, F. Beas, A. Cattani, A. Cortínez, C. Henriquez, A. Gleissner, A. Jara, E. Panteón, R. Youlton, L. Tellerías, I. Totrealba, E. Willshaw, V. Vilheim, por haber derivado pacientes para este estudio.

\section{Referencias}

1. Gelato M., Merriam G: Growth hormone releasing hormone. Ann Rev Physiol 1986; 48: 569-91.
2. Gelato $M$. Malozowsky $S$, Nicolerti $\boldsymbol{M}$ et al.: Growth hormone $(\mathrm{GH})$ responses to Gh-relessing hormone during pubertal development in normal boys and girls: Comparison to idiopathic short stature and GH deficiency. J Clin Endocrinol Metab 1986; 63:1:174-179.

3. Guillemin $R$, Brazeau $P$, Bohlen P, Esch $F:$ Growth hormone releasing factor from a human pancreatic tumor that causes acromegaly. Science 1982; 218: 585-587.

4. Rivier $J$, Spiess $J$, Thorner $M$, Vale $W$ : Characterization of a growth hormone-releasing factor from a human pancreatic islet tumor. Nature 1982: 300: $276-27 \mathrm{~B}$.

5. Takono $K$. Hizuka $N$, Shizume $A$ et al.: Plasma growth hormone response to GH-releasing factor in normal children with short stature and patients with pituitary dwarfism. I Clin Endocrinol Metab 1984; 58 : 236-241.

6. Ragol A, Blizzard $R$, Johanson AJ, Furnatetio $R$ : Growth hormone release in response to human pancreatic tumor growth hormone releasing hormone-40 in children with short stature, J Clin Endocrinol Metso $1984 ; 59$ : $580-586$.

7. Merriom $G R$, Wachter $K W$ : Measurement and analysis of episodic hormone secretion. In: Computers in Endocrinology. Raven Press. New York $1984 ; 325-346$.

8. Smith PJ, Brook GD: Growth hormone releasing hormone or growth hormone treatment in growth hormone insufficiency? Arch Dis Child 1988; 63: 629-634.

9. Thorner MO, Vance AD, Rogol RM et al.: Growth hormone releasing hormone and growth hormone-releasing peptide as potential therapeutic modalities. Review paper. Acta Paediatt Scand (Suppl) $1990 ; 367: 29 \cdot 32$.

10. Smith PJ, Brook CGD: The place of intravenous GHRH $1-40$ studjes in the therapy of growth hormone deficient children with GHRH. Clin Endocrinol (Oxf) 1987; 27: 97-105.

11. Schriok E, Lustig RH, Rosenthal SM, Kaplan SI, Grumbach M: Effect of growth hormone (GH)releasing hormone, on plasma $\mathrm{GH}$ in relation to magnitude and duration of $\mathrm{GH}$ deficiency in 26 children and adults with isolated $G H$ deficiency or multiple pituitary hormone deficiencies: evidence for hypothalamic GRH, deficiency. I Clin Endoctinol Metab 1984;58: 1043-1049. 